\title{
Selection of Database Management System with Fuzzy-AHP for Electronic Medical Record
}

\author{
M. Ebrahimi \\ Faculty of New Sciences and Technologies, University of Tehran, Tehran, Iran \\ E-mail: mo.ebrahimi@ut.ac.ir \\ M. Taheri \\ Faculty of New Sciences and Technologies, University of Tehran, Tehran, Iran \\ E-mail: masoudtaheri@ut.ac.ir
}

\begin{abstract}
Database selection is one of the main problems in designing electronic medical record (EMR) Software, since there are many different data with different types and formats in it. This work provides an approach for selecting suitable database management system (DBMS) with fuzzy analytical hierarchical process that gives capabilities of requirements to electronic medical record software. Criteria are choose based upon capabilities of requirements including supporting from type of operating system and programming language, maximum of table size, indexing, replication and access control. In addition, alternatives are PostgreSQL, MySQL, Oracle, DB2 and Microsoft SQL Server. According to the application of fuzzy analytical hierarchical process a ranking is obtained to decision making the best database management system for electronic medical record software.
\end{abstract}

Index Terms-Electronic Medical Record, Data Base Management System, Software Design, FAHP, FMCDM.

\section{INTRODUCTION}

Electronic medical record is the basis of all the activity in healthcare information technology [1]. Actually, without development of an electronic medical record software which includes health data and information related to humans' population which is available in every time and place for clinics, Hospitals and any healthcare organization, other activities can't be done. Patient records are one of the information resources that contain vast volume of data with many different data types causing the storage system in EMR to face serious challenges $[1,8]$.

In order to solve these challenges and problems, a number of storage approaches and DBMS databases are proposed; each one having specific strengths and weaknesses. Software engineers or designers, according to requirements and capabilities expected from EMR, chooses suitable DBMS. In this work, one of the fuzzy multi criteria decision making (FMCDM) methods is called FAHP which is used to select DBMS. In FAHP, decision maker has a set of alternatives and a set of criteria that appropriate alternative will be chosen according to the pairwise comparison between alternatives and criteria [2].

Hellman and et al [3] have been using AHP to select the appropriate DBMS for Erlang programing language in which PostgreSQL, MySQL, Berkeley DB and Ingres are alternatives and the set of criteria are include Safety, Large Data, Replication, Mint Logical Constraint, and Erlang Interface. The priorities which obtained based on AHP in the work [3] are as follows:

\section{Berkeley $D B<M y S Q L<$ PostgreSQL $<$ Ingres}

In the present study, the criteria are operating system, indexes, access control, size of table, replication and programing language and alternatives are PostgreSQL, MySQL, DB2, Oracle and Microsoft SQL Server.

In fact in the above mentioned work all comparison and all scales are considered as crisp number. To compare different criteria such as, $C_{1}=$ operating system, $C_{2}=$ indexes, $C_{3}=$ access control, $C_{4}=$ size of table, $C_{5}=$ replication, $C_{6}=$ programming language.

It is better to use fuzzy scales and linguistic terms. Therefore to cover the subjective uncurtaining of decision-maker for evaluation of criteria and alternatives we propose FAHP.

Based on our best knowledge, this work is the first study for ranking different DBMS to EMR software based on FAHP. In this work for the first time criteria are considered operating system, indexes, access control, size of table, replication, programming language. In fact the previous work just introduce Safety, Large Data, Replication, Mint Logical Constraint, and Erlang Interface. Furth more PostgreSQL, MySQL, db2, oracle, $S Q L$ are added as alternatives.

\section{Problem Definition}

The problem is defined using a hierarchical structure including goal, number of criteria and alternatives. In other words at the beginning of decision making process, the decision-maker according to alternatives, criteria and objectives implies the relationship between these elements that are expressed in terms of hierarchy. Figure 1 shows the hierarchical scheme or description of 
problem for DBMS selection. As shown in Figure 1, the main goals is selecting the suitable DBMS among PostgreSQL (PSQL), Oracle, MySQL (MSQL), DB2 and $S Q L$ based on the following criteria:
Operating System, Indexes, Access Control, Size of Table, Replication, Language Programming.

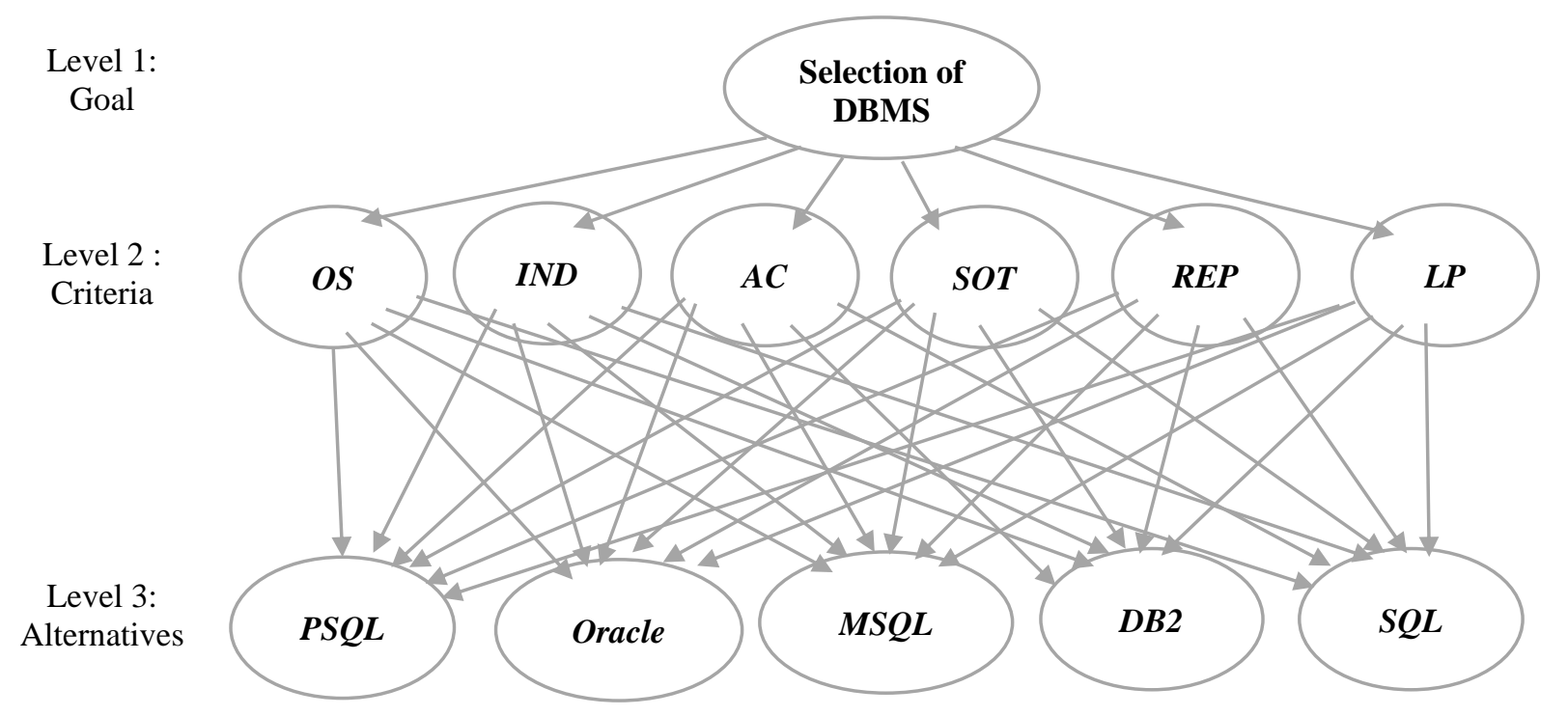

Fig. 1. Hierarchical structure for decision making problem [7]

\section{FUZZY AHP}

One of the multi-criteria decision making techniques, the analytic hierarchical process (AHP), is used widely to assist decision-makers in the industry and has been used for various applications [9, 19]. Saaty first developed the AHP for decision making. Marshall, proposed an AHP methods that had three steps and then a more direct method was adopted for decision making. In this method the determining factor for choosing the suitable case is the one with the highest weight of alternative. [6]. Also, Zahedi offers various applications and sources on the AHP [2].

The fuzzy AHP method is obtained from combination of AHP and fuzzy logic which makes it possible for the decision-maker to make more accurate decisions. Fuzzy set theory is a mathematical theory pioneered by Zadeh, designed to model the uncertainty and imprecision of human cognitive processes [4]. In fact, one of the main points that should be considered in conjunction with fuzzy and crisp concept, is that the entire crisp concept can be extended and expressed using fuzzy concepts. The theory of fuzzy sets and fuzzy logic are used in different types of applications. They are also used to express the concepts more accurately [20]. Among the various applications of fuzzy set theory, design and engineering are considered to be of more significance for various companies and organizations since they lack sufficient data and are faced with more inaccurate concepts. [2].

The key idea of fuzzy set theory is that an element has a degree of membership in a fuzzy set. A fuzzy set is defined by a membership function. The membership function maps elements in the universe of discourse to elements within a certain interval, which is usually $[0,1]$ [5]. Fuzzy concepts are used for doing comparisons between DBMS based on criteria and these concepts are expressed by Linguistic Terms. There are different scoring methods that differ from one another in number of point or linguistic terms such as 5, 7, 9 and 11-point scoring system. And coring system that used in this work is a 9-point scoring method. In this study, triangular fuzzy numbers are used to represent subjective pair-wise comparisons of the selection process (Table1). A triangular fuzzy number denoted as $\widetilde{a}=(b, a, c)$ where $b \leq a \leq c$, has the following triangular-type membership function [11] [18]:

$$
\widetilde{a}(x)=\left\{\begin{array}{lc}
0 & x<b \\
\frac{x-b}{a-b} & b \leq x \leq a \\
\frac{c-x}{c-a} & a \leq x \leq c \\
0 & x>c
\end{array}\right.
$$

Table 1. Linguistic Term and Corresponding fuzzy number [6]

\begin{tabular}{|c|c|}
\hline Linguistic term & Fuzzy Number \\
\hline Equally important & $(1,1,2)$ \\
\hline Moderately more important & $(2,3,4)$ \\
\hline Strongly more important & $(4,5,6)$ \\
\hline Very strongly more important & $(6,7,8)$ \\
\hline Extremely more important & $(8,9,10)$ \\
\hline
\end{tabular}

Alpha-cut concept is used to perform mathematical operations such as multiplication, division and subtraction, which $\alpha$ is value in [0, 1]. Alpha-cut for triangular number is obtained by using the formula 2 : 


$$
\begin{gathered}
\forall \alpha \in[0,1] M_{\alpha}=\left[b^{\alpha}, c^{\alpha}\right]= \\
{[(a-b) \alpha+1,-(c-a) \alpha+c]}
\end{gathered}
$$

Actually, $\alpha$ - cut result is interval and mathematical operation are perfumed on this interval. The main operation for positive fuzzy number such as $\tilde{A}_{1}=$ $\left(b_{1}, a_{1}, c_{1}\right), \tilde{A}_{2}=\left(b_{2}, a_{2}, c_{2}\right)$ are shown in formula 3,4 , 5 and 6 [10] [11]:

$$
\begin{gathered}
\tilde{A}_{1} \oplus \widetilde{A}_{2}=\left(b_{1}+b_{2}, a_{1}+a_{2}, c_{1}+c_{2}\right) \\
\tilde{A}_{1} \otimes \widetilde{A}_{2}=\left(b_{1} * b_{2}, a_{1} * a_{2}, c_{1} * c_{2}\right) \\
\tilde{A}_{1} \ominus \widetilde{A}_{2}=\left(b_{1}-c_{2}, a_{1}-a_{2}, c_{1}-b_{2}\right) \\
\tilde{A}_{1} \oslash \widetilde{A}_{2}=\left(b_{1} / c_{2}, a_{1} / a_{2}, c_{1} / b_{2}\right)
\end{gathered}
$$

The selection steps of suitable alternative by using FAHP can be summarized as follows:

\section{Step 1. Problem definition:}

At first, alternative and criteria are identified and they are shown in the hierarchical diagram. For this work hierarchical diagram of problem definition is shown in figure 1. In this figure the first level represents the main goal of the work, the second level shows criteria and the last level shows the alternative.

\section{Step 2. Matrix of pairwise comparison criteria:}

This step has a Matrix in which the importance of each criteria, relative to one another is expressed by linguistic terms, and the entries of matrix are filled relatively. The linguistic terms used in this work are demonstrated in table 1 . In this step for any of the criteria, eigenvalue is computed and the obtained eigenvalue shows the weight of the criteria.

Step 3. Incorporation of pairwise comparisons of alternatives with respect to criteria in a matrix:

At this step, number of matrix is equal to the number of criteria and all of the alternatives are to be compared with each other based on all of the criteria.

\section{Step 4. Calculation of eigenvalue for all matrixes:}

For each matrix in Step 2 and 3, the eigenvalues are calculated and as a result the first for all rows, the geometric mean is calculate by the formula number 7 and then for any matrix, the obtained values are normalized and placed in a separate column.

Step 5. Selecting the suitable alternative (determining the priority of alternatives):

Finally, the priority of alternatives for selecting the best choice are determined. In order to do this, the vector weight obtained in step 2 is multiplied by all of the eigenvalues in Step 3.

\section{PAIRWISE COMPARISON OF CRITERIA}

In the present study six criteria are considered as follows:

- Different operating systems that the database can be applied on them: In this criteria, more operating systems for a DBMS means less importance regarding the selection of a operating system. In the comparison tables this criteria is abbreviated as $C_{1}=O S$.

- Number of indexing methods that the database can support: more methods of indexing for a DBMS means more importance regarding the topic of indexing. This criteria is shown with $C_{2}=I N D$ in the comparison tables.

- Number of access control methods that database provides: more access control methods for a DBMS means more importance regarding the topic of access control. In the FAHP algorithm access control is abbreviated with $C_{3}=A C$.

- Maximum size of a table that can be created in the database: larger table size in the database shows the importance regarding the topic of supporting larger data. We abbreviated this criteria as $C_{4}=$ SOT.

- Different type of mechanisms for replication: more mechanisms of replication in a DBMS means higher importance of replication. In the comparison tables this criteria is abbreviated as $C_{5}=R E P$

- Programming languages that can be supported: more programming languages which a DBMS can be configured by show less importance regarding the selection of the programming language in it. Abbreviation for this value is $C_{5}=L A N$.

At first, criteria matrix is created. In this matrix, fuzzy number is allocated to entries based on analysis relative to expected feature from EMR software. For example, one of the important features that the software should have, is the support for large amounts of data. Therefore the importance of information or data replication and capability to implement distributed data is far more than the type of operating system or programming language by which the software works. So, priority of replication relative to operating system can be expressed as extremely more important and as a result fuzzy number that placed in $(R E P, O S)$ is $(8,9,10)$, and its inverse i.e. $(1 / 10,1 / 8,1 / 9)$, placed in $(O S, R E P)$. Similarly, another entries are all set. Matrix of criteria comparison is shown in table 2 and the last column of the matrix represents eigenvalue and as seen Eigenvalue in the criteria matrix is demonstrative of weight of criteria.

\section{PAIRWISE COMPARISON OF ALTERNATIVES BASED ON CRITERIA}

As stated before, the purpose is selecting the suitable database for EMR software. All alternatives together are compared on the basis of criteria. 


\section{A. Matrix For Comparing Alternatives Based On OS}

Relative information to create this matrix from [7] are taken. The number of operating systems that are supported by each DBMS, value of relative comparison and eigenvalue for any rows are shown in table 2. For example the number of OS for Oracle is 5 and for $S Q L$ is 1. Therefore, degree of $O S$ criteria importance for $S Q L$ compared to Oracle is expressed with strongly more important (Table 1) and $(4,5,6),(1 / 6,1 / 5,1 / 6)$ are placed in $(S Q L$, Oracle) and (Oracle, $S Q L)$.

\section{B. Matrix For Comparing Alternatives Based On Indexes}

According to information available in [7], different type of indexing methods that any DBMS supports are

Table 2. Matrix of pairwise comparison of criteri

\begin{tabular}{|c|c|c|c|c|c|c|c|}
\hline criteria & OS & IND & AC & SOT & REP & LP & Eigenvalue \\
\hline OS & $(1,1,2)$ & $(1 / 10,1 / 9,1 / 8)$ & $(1 / 8,1 / 7,1 / 6)$ & $(1 / 8,1 / 7,1 / 6)$ & $(1 / 10,1 / 9,1 / 8)$ & $(1,1,2)$ & $(0 / 018,0 / 024,0 / 037)$ \\
\hline IND & $(8,9,10)$ & $(1,1,2)$ & $(4,5,6)$ & $(2,3,4)$ & $(1 / 8,1 / 7,1 / 6)$ & $(8,9,10)$ & $(0 / 154,0 / 226,0 / 334)$ \\
\hline AC & $(6,7,8)$ & $(1 / 6,1 / 5,1 / 4)$ & $(1,1,2)$ & $(4,5,6)$ & $(1 / 8,1 / 7,1 / 6)$ & $(8,9,10)$ & $(0 / 097,0 / 138,0 / 159)$ \\
\hline SOT & $(6,7,8)$ & $(1 / 4,1 / 3,1 / 2)$ & $(1 / 6,1 / 5,1 / 4)$ & $(1,1,2)$ & $(1 / 6,1 / 5,1 / 4)$ & $(6,7,8)$ & $(0 / 061,0 / 089,0 / 138)$ \\
\hline REP & $(8,9,10)$ & $(6,7,8)$ & $(6,7,8)$ & $(4,5,6)$ & $(1,1,2)$ & $(8,9,10)$ & $(0 / 553,0 / 448,0 / 716)$ \\
\hline LP & $(1,1,2)$ & $(1 / 10,1 / 9,1 / 8)$ & $(1 / 10,1 / 9,1 / 8)$ & $(1 / 8,1 / 7,1 / 6)$ & $(1 / 10,1 / 9,1 / 8)$ & $(1,1,2)$ & $(0 / 017,0 / 023,0 / 036)$ \\
\hline
\end{tabular}

Table 3. Matrix for compared alternative based on Operating System [7] [12] [13] [14] [15] [16] [17]

\begin{tabular}{|c|c|c|c|c|c|c|c|}
\hline OS & PSQL & Oracle & MSQL & DB2 & SQL & $\begin{array}{c}\text { Number } \\
\text { of OS }\end{array}$ & Eigenvalue \\
\hline PSQL & $(1,1,2)$ & $(1 / 4,1 / 3,1 / 2)$ & $(2,3,4)$ & $(1,1,2)$ & $(1 / 8,1 / 7,1 / 6)$ & 7 & $(0.054,0.064,0.1)$ \\
\hline Oracle & $(2,3,4)$ & $(1,1,2)$ & $(4,5,6)$ & $(1,1,2)$ & $(1 / 6,1 / 5,1 / 4)$ & 5 & $(0.1,0.118,0.179)$ \\
\hline MSQL & $(1 / 4,1 / 3,1 / 2)$ & $(1 / 6,1 / 5,1 / 4)$ & $(1,1,2)$ & $(1 / 4,1 / 3,1 / 2)$ & $(1 / 10,1 / 9,1 / 8)$ & 9 & $(0.024,0.028,0.041)$ \\
\hline DB2 & $(1,1,2)$ & $(1,1,2)$ & $(2,3,4)$ & $(1,1,2)$ & $(1 / 6,1 / 5,1 / 4)$ & 6 & $(0.076,0.085,0.143)$ \\
\hline SQL & $(6,7,8)$ & $(4,5,6)$ & $(8,9,10)$ & $(4,5,6)$ & $(1,1,2)$ & 1 & $(0.358,0.413,0.535)$ \\
\hline
\end{tabular}

Shown and can be compared alternatives based on indexes. Matrix of this comparison is showed in table 4. As stated before, in a DBMS supporting more indexing methods, the importance of indexing is higher. For example, PostgreSQL supports 10 methods for indexing, while the number of indexing methods in MySQL is 1 . Therefore, indexing in PostgreSQL is expressed by extremely more important than $M y S Q L$. Also importance of indexing in DB2 and SQL is equal. So, (PSQL, MySQL) $=(8,9,10)$ and $(D B 2, M y S Q L)=(1,1,2)$.

\section{Matrix For Comparing Alternatives Based On Access Control}

More access control methods in a DBMS means more importance regarding the topic of access control in a DBMS. For example, total access control methods in PostgreSQL, Oracle and SQL are equal to 9, therefore the importance of access control in these three are equal. On the other hand, in $M y S Q L$, number of access control methods is 4. So, importance of access control in PostgreSQL, Oracle and SQL compared to MySQL is can be expressed by very strongly more important. Therefor (Oracle, $S Q L)$ and $(M y S Q L$, Oracle) equals to $(1,1,2)$, $(1 / 8,1 / 7,1 / 6)$. Other entries are filled according to this analysis (Table 5).

Table 4. Matrix for compared alternative based on Indexes [7] [12] [13] [14] [15] [16] [17]

\begin{tabular}{|c|c|c|c|c|c|c|c|}
\hline IND & PSQL & Oracle & MSQL & DB2 & SQL & $\begin{array}{c}\text { Number of } \\
\text { Indexes } \\
\text { approach }\end{array}$ & Eigenvalue \\
\hline PSQL & $(1,1,2)$ & $(2,3,4)$ & $(8,9,10)$ & $(6,7,8)$ & $(6,7,8)$ & 10 & $(0.327,0.529,0.81)$ \\
\hline Oracle & $(1 / 4,1 / 3,1 / 2)$ & $(1,1,2)$ & $(6,7,8)$ & $(4,5,6)$ & $(4,5,6)$ & 8 & $(0.173,0.283,0.445)$ \\
\hline MSQL & $(1 / 10,1 / 9,1 / 8)$ & $(1 / 8,1 / 7,1 / 6)$ & $(1,1,2)$ & $(1 / 6,1 / 5,1 / 4)$ & $(1,1,2)$ & 1 & $(0.026,0.033,0.445)$ \\
\hline DB2 & $(1 / 8,1 / 7,1 / 6)$ & $(1 / 6,1 / 5,1 / 4)$ & $(4,5,6)$ & $(1,1,2)$ & $(1,1,2)$ & 4 & $(0.055,0.085,0.146)$ \\
\hline SQL & $(1 / 8,1 / 7,1 / 6)$ & $(1 / 6,1 / 5,1 / 4)$ & $(1,1,2)$ & $(1,1,2)$ & $(1,1,2)$ & 4 & $(0.042,0.061,0.12)$ \\
\hline
\end{tabular}

Table 5. Matrix for compared alternative based on Access Control [7] [12] [13] [14] [15] [16] [17]

\begin{tabular}{|c|c|c|c|c|c|c|c|}
\hline AC & PSQL & Oracle & MSQL & DB2 & SQL & $\begin{array}{c}\text { Number of } \\
\text { Access } \\
\text { Control } \\
\text { approach }\end{array}$ & Eigenvalue \\
\hline PSQL & $(1,1,2)$ & $(2,3,4)$ & $(4,5,6)$ & $(8,9,10)$ & $(4,5,6)$ & 9 & $(0.293,0.5,0.81)$ \\
\hline Oracle & $(1 / 4,1 / 3,1 / 2)$ & $(1,1,2)$ & $(2,3,4)$ & $(6,7,8)$ & $(2,3,4)$ & 9 & $(0.138,0.25,0.435)$ \\
\hline MSQL & $(1 / 6,1 / 5,1 / 4)$ & $(1 / 4,1 / 3,1 / 2)$ & $(1,1,2)$ & $(4,5,6)$ & $(1,1,2)$ & 4 & $(0.007,0.109,0.205)$ \\
\hline DB2 & $(1 / 10,1 / 9,1 / 8)$ & $(1 / 8,1 / 7,1 / 6)$ & $(1 / 6,1 / 5,1 / 4)$ & $(1,1,2)$ & $(1 / 6,1 / 5,1 / 4)$ & 8 & $(0.019,0.031,0.050)$ \\
\hline SQL & $(1 / 6,1 / 5,1 / 4)$ & $(1 / 4,1 / 3,1 / 2)$ & $(1,1,2)$ & $(4,5,6)$ & $(1,1,2)$ & 9 & $(0.067,0.109,0.205)$ \\
\hline
\end{tabular}




\section{Matrix For Comparing Alternatives Based On Size Of Table}

Maximum size of a table for DBMS can be obtained in [7]. Importance of supporting the large data in a database is more important if it is to be a large table. Results of the comparison and calculation of eigenvalue are shown in table 6. For example, maximum size of table in Oracle is very more than $D B 2$, and importance of supporting large data in $D B 2$ compared to Oracle is expressed by extremely more important, therefore (DB2, Oracle $)=(8,9,10)$.

\section{E. Matrix For Comparing Alternatives Based On Replication}

Replication methods for each alternative is demonstrated in [7]. Number of methods that DBMS can support for replication, comparisons between alternative and eigenvalue for each row are shown in table 7. More replication methods shows more importance regarding the importance of replication in a DBMS. For example PostgreSQL and DB2 used one method for replication while $M y S Q L$ used three methods, therefor $(D B 2, M y S Q L)$ $=(1 / 6,1 / 5,1 / 4)$ and $(D B 2$, PostgreSQL $)=(1,1,2)$.

Table 6. Matrix for compared alternative based on Size of Table [7] [12] [13] [14] [15] [16] [17]

\begin{tabular}{|c|c|c|c|c|c|c|c|}
\hline SOT & PSQL & Oracle & MSQL & DB2 & SQL & $\begin{array}{c}\text { Maximum } \\
\text { of Table } \\
\text { Size }\end{array}$ & Eigenvalue \\
\hline PSQL & $(1,1,2)$ & $(2,3,4)$ & $(1 / 6,1 / 5,1 / 4)$ & $(1 / 10,1 / 9,1 / 8)$ & $(2,3,4)$ & $32 \mathrm{~TB}$ & $(0.054,0.097,0.162)$ \\
\hline Oracle & $(1 / 4,1 / 3,1 / 2)$ & $(1,1,2)$ & $(1 / 6,1 / 5,1 / 4)$ & $(1 / 10,1 / 9,1 / 8)$ & $(1 / 8,1 / 7,1 / 6)$ & $4 \mathrm{~GB}$ & $(0.021,0.034,0.06)$ \\
\hline MSQL & $(4,5,6)$ & $(4,5,6)$ & $(1,1,2)$ & $(1 / 6,1 / 5,1 / 4)$ & $(1 / 4,1 / 3,1 / 2)$ & $256 \mathrm{~TB}$ & $(0.091,0.149,0.252)$ \\
\hline DB2 & $(8,9,10)$ & $(8,9,10)$ & $(4,5,6)$ & $(1,1,2)$ & $(2,3,4)$ & $2 \mathrm{ZB}$ & $(0.343,0.558,0.886)$ \\
\hline SQL & $(1 / 4,1 / 3,1 / 2)$ & $(6,7,8)$ & $(2,3,4)$ & $(1 / 4,1 / 3,1 / 2)$ & $(1,1,2)$ & $524272 \mathrm{~TB}$ & $(0.093,0.159,0.283)$ \\
\hline
\end{tabular}

Table 7. Matrix for compared alternative based on Replication [7] [12] [13] [14] [15] [16] [17]

\begin{tabular}{|c|c|c|c|c|c|c|c|}
\hline REP & PSQL & Oracle & MSQL & DB2 & SQL & $\begin{array}{c}\text { Number of } \\
\text { Replication } \\
\text { approach }\end{array}$ & Eigenvalue \\
\hline PSQL & $(1,1,2)$ & $(1 / 4,1 / 3,1 / 2)$ & $(1 / 6,1 / 5,1 / 4)$ & $(1,1,2)$ & $(1,1,2)$ & 1 & $(0.052,0.058,0.186)$ \\
\hline Oracle & $(2,3,4)$ & $(1,1,2)$ & $(1 / 4,1 / 3,1 / 2)$ & $(2,3,4)$ & $(2,3,4)$ & 2 & $(0.113,0.228,0.427)$ \\
\hline MSQL & $(4,5,6)$ & $(2,3,4)$ & $(1,1,2)$ & $(4,5,6)$ & $(2,3,4)$ & 3 & $(0.226,0.434,0.761)$ \\
\hline DB2 & $(1,1,2)$ & $(1 / 4,1 / 3,1 / 2)$ & $(1 / 6,1 / 5,1 / 4)$ & $(1,1,2)$ & $(1 / 4,1 / 3,1 / 2)$ & 1 & $(0.039,0.068,0.14)$ \\
\hline SQL & $(2,3,4)$ & $(1,1,2)$ & $(1 / 4,1 / 3,1 / 2)$ & $(2,3,4)$ & $(1,1,2)$ & 2 & $(0.098,0.183,0.372)$ \\
\hline
\end{tabular}

F. Matrix of Comparing Alternatives Based On Programming Language

Programming languages that each DBMS supports, are mentioned in [7]. Number of programming languages that DBMS supports, comparison between alternatives and eigenvalues are shown in table 8. More supported programming languages in a DBMS shows less importance regarding the topic in a DBMS. For example, Oracle supports 32 languages, but $S Q L$, on the other hand supports 6 languages. Therefor the programming language in $\mathrm{SQL}$ is expressed with extremely more important and therefore: $(S Q L$, Oracle $)=(8,9,10)$, $($ Oracle, $S Q L)=(1 / 10,1 / 9,1 / 8)$.

\section{RESULTS}

Overall, alternative priority for selecting suitable DBMS is obtained by multiplication between weight vectors (eigenvalues) of criteria pairwise comparison and any eigenvalue rows of alternative pairwise comparison. In order to do dfuzzy, Middle value in fuzzy number has been considered as the crisp number for it.

Table 8. Matrix of compared alternative based on Programming Language [7] [12] [13] [14] [15] [16] [17]

\begin{tabular}{|c|c|c|c|c|c|c|c|}
\hline LP & PSQL & Oracle & MSQL & DB2 & SQL & $\begin{array}{c}\text { Number of } \\
\text { Programming } \\
\text { Language }\end{array}$ & Eigenvalue \\
\hline PSQL & $(1,1,2)$ & $(8,9,10)$ & $(6,7,8)$ & $(4,5,6)$ & $(1,1,2)$ & 7 & $(0.251,0.394,0.627)$ \\
\hline Oracle & $(1 / 10,1 / 9,1 / 8)$ & $(1,1,2)$ & $(1 / 6,1 / 5,1 / 4)$ & $(1 / 8,1 / 7,1 / 6)$ & $(1 / 10,1 / 9,1 / 8)$ & 23 & $(0.016,0.025,0.036)$ \\
\hline MSQL & $(1 / 8,1 / 7,1 / 6)$ & $(4,5,6)$ & $(1,1,2)$ & $(1 / 6,1 / 5,1 / 4)$ & $(1 / 8,1 / 7,1 / 6)$ & 17 & $(0.035,0.052,0.084)$ \\
\hline DB2 & $(1 / 6,1 / 5,1 / 4)$ & $(6,7,8)$ & $(4,5,6)$ & $(1,1,2)$ & $(1 / 6,1 / 5,1 / 4)$ & 11 & $(0.081,0.133,0.198)$ \\
\hline SQL & $(1,1,2)$ & $(8,9,10)$ & $(6,7,8)$ & $(4,5,6)$ & $(1,1,2)$ & 6 & $(0.251,0.394,0.627)$ \\
\hline
\end{tabular}

$\left(\begin{array}{cccccc}0.064 & 0.529 & 0.5 & 0.097 & 0.085 & 0.394 \\ 0.118 & 0.283 & 0.25 & 0.034 & 0.228 & 0.025 \\ 0.028 & 0.399 & 0.109 & 0.149 & 0.434 & 0.052 \\ 0.085 & 0.85 & 0.031 & 0.558 & 0.068 & 0.133 \\ 0.061 & 0.061 & 0.109 & 0.159 & 0.183 & 0.394\end{array}\right) *\left(\begin{array}{l}0.024 \\ 0.226 \\ 0.138 \\ 0.089 \\ 0.448 \\ 0.023\end{array}\right)=\left(\begin{array}{l}0.3148 \\ 0.2443 \\ 0.2070 \\ 0.1439 \\ 0.1087\end{array}\right)$


Table 9. Finally priority of Database Management System

\begin{tabular}{|c|c|c|c|c|}
\hline MySQL & PostgreSQL & Oracle & SQL & DB2 \\
\hline 0.3148 & 0.2443 & 0.2070 & 0.1439 & 0.1087 \\
\hline
\end{tabular}

Finally priority that obtained is shown in table 10 as:

$$
D B 2<S Q L<\text { Oracle }<\text { PostgreSQL }<M y S Q L
$$

As previously mentioned, purpose of this paper is the selection of suitable DBMS for electronic medical record with fuzzy AHP based on functionality requirement. According to alternatives and criteria considered and analyses performed by FAHP, the suitable DBMS for EMR is $M Y S Q L$.

\section{CONCLUSION}

The present study successfully applies a multi criteria decision making model based on fuzzy analytic hierarchy process to select the best choice of database management system (DBMS) that gives capabilities of requirements to electronic medical record software. In the second level of the hierarchical structure six main criteria are considered. These main criteria are chosen based upon capabilities of requirements including supporting from type of operating system and programming language, maximum of table size, indexing, replication and access control. Furthermore in the third level of the decision making model five different alternatives PostgreSQL, MySQL, Oracle, DB2 and Microsoft SQL Server, are considered. Owing to the application of FAHP a ranking is obtained to select the best database management system for electronic medical record software.

\section{REFERENCES}

[1] K. A.WAGER, F. WICKHAM L.EE and J. P.GLASER, HEALTH CARE INFORMATION SYSTEMS, A Practical Approach for Health Care Management, San Francisco: Jossy-Bass, 2009.

[2] Z. AYA G, "A fuzzy AHP-based simulation approach to concept evaluation in a NPD environment," Department of Industrial Engineering, Istanbul K"ult"ur University, 2004. R. Nicole, "Title of paper with only first word capitalized", J. Name Stand. Abbrev., in press.

[3] E. Hellman, W. E. Sullivan and F. Cesarini, "Evaluation of Database Management Systems for Erlang," 2006. M. Young, The Technical Writer's Handbook. Mill Valley, CA: University Science, 1989.

[4] F. Lootsma, "Fuzzy Logic for Planning and Decision Making Kluwer, Dordrecht," The Netherlands.

[5] C. Negoita, "Expert Systems and Fuzzy Systems," Benjamin/Cummings,, 1985.

[6] T. Saaty, "Decision making, scaling, and number crunching," 1989, p. 404-409.

[7] http://db-engines.com/en/2014-10-17.

[8] J. Yang, D. Tang, Y. Zhou, A Distributed Storage Model for HER Based on HBase", International Conference Information Management, Innovation Management and Industries Engineering 2011, College of Medical Information Engineering, Guangzhou, Chin.
[9] M. Scott, "Quantifying certainty in design decisions: examining AHP, in Proceedings" DETC 2002.

[10] A. Kaufmann and M. M. Gupta, " introduction to Fuzzy Arithmetic: Theory and Application", Van Nostrand Reinhold, New York, NY (1985).

[11] T. Yu-Cheng, J.B.Malcolm, "Application and Development of a Fuzzy Analytic Hierarchy Proces within a Capital Investment study", Journal of Economics and Management, 2005, Vol. 1, No. 2, 207-230.

[12] https://www.digitalocean.com/community/tutorials/sqlitevs-mysql-vs-postgresql-a-comparison-of-relationaldatabase-management-systems/2014-10-13.

[13] http://www.mysql.com/2014-10-13.

[14] http://www.postgresql.com/2014-10-15.

[15] http://www.oracle.com/2014-10-17.

[16] http://www-01.ibm.com/software/data/db2/2014-10-17.

[17] http://www.sqlservercentral.com/2014-10-13.

[18] Debmallya Chatterjee et al. "Study of Fuzzy-AHP Model To Search The Criterion In The Evaluation Of The Best Technical Institutions: A Case Study", International Journal of Engineering Science and Technology, Vol. 2(7), 2010, 2499-2510.

[19] Renuka Nagpal and et all, " Rank University Websites Using Fuzzy AHP and Fuzzy TOPSIS Approach on Usability ", I. J. Information Engineering and Electronic Business,2015,1,29-36, Published Online January 2015 in MECS, ijieeb.2015.01.04.

[20] F.Jinghong, W.Yan and Y.Hongcan, "The Application of Database Analytic Hierarchy Process (AHP) in Teacherto-Teacher Assessment", I. J. Information Engineering and Electronic Business, IJEME Vol. 3, No. 1, January 2013.

\section{Authors' Profiles}

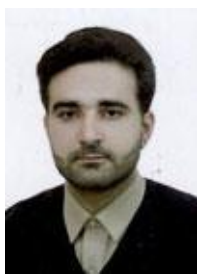

Dr Morteza Ebrahimi is an assistant professor in Applied Mathematics. Also, $\mathrm{He}$ has been a staff in the University of Tehran, Faculty of New Sciences and Technologies. His research expertise is in multi criteria decision making, networked systems, mathematical modeling of diffusion problems that are arisen in the fields of heat transfer, food, social networks and human respiration. Dr Ebrahimi has been active in the combined numerical-probabilistic algorithm based on Monte Carlo methods for solving inverse problems and integral equations. He has published numerous research articles, more than 48 in peer-refereed journals and proceedings.

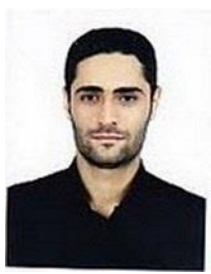

Masoud B. Taheri is Master of Healthcare Information Technology Engineering University of Tehran, Faculty of New Sciences and Technologies. His area of interest include Database, Distributed Database, Cloud Computing, NoSQL Storage Methods, EMR/EHR Software and Software development. 